\title{
FTIR and Electrical Study of Dysprosium Doped Cobalt Ferrite Nanoparticles
}

\author{
Hemaunt Kumar, ${ }^{1}$ Jitendra Pal Singh, ${ }^{2}$ R. C. Srivastava, ${ }^{1}$ \\ P. Negi, ${ }^{1}$ H. M. Agrawal, ${ }^{1}$ and K. Asokan ${ }^{3}$ \\ ${ }^{1}$ Department of Physics, G. B. Pant University of Ag. \& Technology, Pantnagar, Uttarakhand-263145, India \\ ${ }^{2}$ Department of Applied Science, Krishna Engineering College, Mohan Nagar, Ghaziabad, Uttar Pradesh-201007, India \\ ${ }^{3}$ Inter University Accelerator Centre, New Delhi 110067, India \\ Correspondence should be addressed to Hemaunt Kumar; hvatsal@gmail.com
}

Received 9 January 2014; Revised 7 April 2014; Accepted 7 April 2014; Published 18 May 2014

Academic Editor: Tian Xia

Copyright (C) 2014 Hemaunt Kumar et al. This is an open access article distributed under the Creative Commons Attribution License, which permits unrestricted use, distribution, and reproduction in any medium, provided the original work is properly cited.

We have studied the role of $\mathrm{Dy}^{3+}$ doping on the XRD, TEM, FTIR, and dielectric and electrical properties of $\mathrm{CoFe}_{2} \mathrm{O}_{4}$ at room temperature. Cubic spinel phase of $\mathrm{CoFe}_{2-x} \mathrm{Dy}_{x} \mathrm{O}_{4}(x=0.00,0.05,0.10$, and 0.15$)$ was synthesized by using different sintering temperatures $\left(300,500,700\right.$, and $\left.900^{\circ} \mathrm{C}\right)$. The two absorption bands $v_{1}$ and $v_{2}$ are observed in Fourier transform infrared spectroscopy (FTIR) spectra corresponding to the tetrahedral and octahedral sites, which show signature of spinel structure of the sample. For the sample sintered at $300^{\circ} \mathrm{C}$, the dielectric constant is almost unchanged with the frequency at the particular concentrations of $x=0.00$ and 0.05 . Similar result is obtained for the sample sintered at $500^{\circ} \mathrm{C}(x=0.10,0.15), 700^{\circ} \mathrm{C}(x=0.05,0.10$, and 0.15$)$, and $900^{\circ} \mathrm{C}(x=0.05,0.10)$. An increase in the dielectric constant was observed for the undoped cobalt ferrite sintered at 500,700 , and $900^{\circ} \mathrm{C}$. The values of electrical resistivity of the materials vary from $\sim 10^{5}$ to $10^{9} \Omega-\mathrm{cm}$.

\section{Introduction}

Spinel ferrites have general formula $\mathrm{MO}: \mathrm{Fe}_{2} \mathrm{O}_{3}$ where $\mathrm{M}$ is $\mathrm{Zn}^{2+}, \mathrm{Ni}^{+2}, \mathrm{Co}^{+2}$, and so forth. It constitutes an important class of magnetic materials having several technological applications like spintronics, magnetic diagnostic, magnetic drug delivery, storage devices, electrical generators, microwave devices, and so forth [1]. Nanoferrites exhibit unusual electrical, magnetic, and optical properties which are sensitive to their structure, method of synthesis, particle size, and type of dopant ions. These materials crystallize into a cubic closed-packed structure of oxygen ions. The cations occupy two types of interstitial sites known as tetrahedral (A) site and octahedral (B) site. The site occupancy is often depicted by the chemical formula $\left(\mathrm{M}^{2+}{ }_{1-\delta} \mathrm{Fe}^{3+}{ }_{\delta}\right)\left[\mathrm{M}^{2+}{ }_{\delta} \mathrm{Fe}^{3+}{ }_{2-\delta}\right] \mathrm{O}^{2-}{ }_{4}$, where the parentheses and square brackets denote $\mathrm{A}$ and $\mathrm{B}$ sites, respectively, $\mathrm{M}$ represents a divalent cation, and $\delta$ is inversion parameter [2].
Among spinel ferrites, $\mathrm{CoFe}_{2} \mathrm{O}_{4}$ is an interesting magnetic material due to its high coercivity $(\sim 5400 \mathrm{Oe})$ and moderate saturation magnetization $(\sim 80 \mathrm{emu} / \mathrm{g})$ as well as remarkable chemical stability and mechanical hardness, which make it a good candidate for the recording media $[3,4]$. The coexistence of spontaneous polarization and magnetization in $\mathrm{CoFe}_{2} \mathrm{O}_{4}$ based composite and core shell nanoparticles is of great importance [5]. However, synthesising composites and core-shell particles is a complicated task rather than synthesizing single phase materials. Doping of $\mathrm{Zn}$ in the cobalt ferrite matrix leads to the increase of dielectric constant, whereas nickel substitution leads to the decrement in the dielectric constant [6]. The signature of ferroelectricity in the magnetically ordered Mo-doped $\mathrm{CoFe}_{2} \mathrm{O}_{4}$ has been investigated and it shows the coexistence of both ferroelectricity and magnetic ordering at room temperature [7]. The effect of $\mathrm{La}^{3+}$ doping on the electrical, dielectric, and magnetic properties of cobalt ferrite prepared by coprecipitation technique has been investigated [8] and 
TABLE 1: Calculated XRD parameters of the samples $\mathrm{CoFe}_{2-x} \mathrm{Dy}_{x} \mathrm{O}_{4}$ sintered at $500^{\circ} \mathrm{C}$.

\begin{tabular}{lcccc}
\hline Samples & $\begin{array}{c}\text { Crystallite size } \\
( \pm 1 \mathrm{~nm})\end{array}$ & $\begin{array}{c}\text { Lattice parameter } \\
( \pm 0.01 \AA)\end{array}$ & $\begin{array}{c}\text { X-ray density } \\
\left( \pm 0.02 \mathrm{gm} / \mathrm{cm}^{3}\right)\end{array}$ & $\begin{array}{c}\text { Specific surface area } \\
\left( \pm 1 \mathrm{~m}^{2} / \mathrm{gm}\right)\end{array}$ \\
\hline$x=0.00$ & 22 & 8.37 & 5.33 & 51 \\
$x=0.05$ & 16 & 8.37 & 5.44 & 69 \\
$x=0.10$ & 13 & 8.36 & 5.57 & 83 \\
$x=0.15$ & 11 & 8.36 & 5.69 & 96 \\
\hline
\end{tabular}

low values of dielectric constant, dielectric loss, and magnetic loss could be correlated with better compositional stoichiometry and Fe ions concentration. The structural, electrical, and magnetic studies of $\mathrm{Gd}^{3+}$ doped cobalt ferrite nanoparticles were investigated and these materials show low loss, high resistivity, and soft magnetic properties due to $\mathrm{Gd}^{3+}$ doping [9].

It was observed that rare earth doped ferrite exhibits better properties; however, limited solubility of these ions in ferrite is a major issue. Moreover, the solubility limit depends on the method of synthesis [8]. In the lanthanide series the magnetic moment varies from $0\left(\mathrm{La}^{3+}\right)$ to $10.5 \mu \mathrm{B}\left(\mathrm{Dy}^{3+}\right)$ and lanthanide ions can be isotropic or anisotropic due to the large variation of the f-electron orbital contribution to the magnetic interaction. The magnetic properties of rare earth doped ferrites are not well understood either in terms of the magnetic moment of the dopant cations or the unpaired $f$ electrons [10]. Among the lanthanide series $\mathrm{Dy}^{3+}$ ion has the highest value of magnetic moment which affects the magnetic and electrical properties of the materials. There are several issues related to the rare earth ions doping in ferrites such as limited solubility of rare earth ions and its effect on the electrical and magnetic properties which are not well understood. Hence, the present work is carried out to get an insight of the underlying physics by performing XRD, TEM, Fourier transform infrared spectroscopy (FTIR), and dielectric and electrical resistivity measurements of $\mathrm{Dy}^{3+}$ doped ferrite.

\section{Experimental}

Nanoparticles of dysprosium doped cobalt ferrite were synthesized by using the chemical route. The stoichiometric amounts of $\mathrm{Fe}\left(\mathrm{NO}_{3}\right)_{3} \cdot 9 \mathrm{H}_{2} \mathrm{O}, \mathrm{Co}\left(\mathrm{NO}_{3}\right)_{2} \cdot 6 \mathrm{H}_{2} \mathrm{O}$, and $\mathrm{Dy}\left(\mathrm{NO}_{3}\right)_{3} \cdot 5 \mathrm{H}_{2} \mathrm{O}$ were dissolved in the distilled water. In the aqueous salt solution, citric acid solution was added with the cations to citric acid molar ratio of $1: 2$. The solution was heated at $85^{\circ} \mathrm{C}$ under constant magnetic stirring until the solution got converted into a viscous gel and was allowed to cool at room temperature. The cooled gel was dried in an oven at $100^{\circ} \mathrm{C}$ for overnight to form the precursor material. The precursor material was sintered at 300, 500, 700, and $900^{\circ} \mathrm{C}$ for $2 \mathrm{hrs}$ to get the nanoparticles of dysprosium doped cobalt ferrite. X-ray diffraction data were collected on Bruker D8 ADVANDED X-ray diffractometer using $\mathrm{Cu} \mathrm{K}_{\alpha}$ radiation $(\lambda=1.54178 \AA)$. The ferrite powder was pressed into pellets of $10 \mathrm{~mm}$ diameter at a presser of 5 ton after mixing with about $2 \%$ by weight of polyvinyl alcohol binder. These pellets

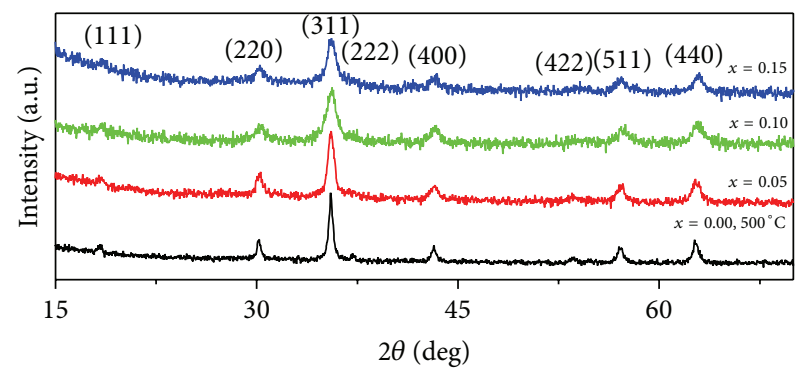

Figure 1: XRD patterns of $\mathrm{CoFe}_{2-x} \mathrm{Dy}_{x} \mathrm{O}_{4}(x=0.00,0.05,0.10 \&$ $0.15)$ sintered at temperature of $500^{\circ} \mathrm{C}$.

were heated in a furnace at $200^{\circ} \mathrm{C}$ for $2 \mathrm{hrs}$. These pellets were polished and coated with silver on both surfaces for dielectric measurements. The dielectric properties of the samples were measured with an Agilent 4285A precision LCR meter in the frequency range of $75 \mathrm{kHz}$ to $5 \mathrm{MHz}$. Fourier transform infrared spectroscopy (FTIR) was recorded at room temperature in the range of 200 to $4000 \mathrm{~cm}^{-1}$ using Bruker make Vortex 70 spectrometer.

\section{Results and Discussion}

3.1. XRD Study. Figure 1 shows the representative $\mathrm{X}$-ray diffraction patterns of $\mathrm{CoFe}_{2-x} \mathrm{Dy}_{x} \mathrm{O}_{4}(x=0.00,0.05,0.10$, and 0.15 ) samples sintered at $500^{\circ} \mathrm{C}$. The synthesized samples exhibit the presence of cubic spinel phase (JCPDS number -03-0864). No impurity phase was detected even for the highest concentration of $\mathrm{Dy}^{3+}(x=0.15)$. All the XRD peaks could be indexed to Fd3m space group with cubic symmetry. It is observed that the XRD peaks of the samples appear to be broadened with increasing $\mathrm{Dy}^{3+}$ concentration. The XRD parameters such as crystallite size, lattice parameters, X-ray density, and specific surface area are tabulated in Table 1. The higher concentration of $\mathrm{Dy}^{3+}$ led to lower crystallinity of the all the samples. This may be because more dopant concentration leads to a higher potential barrier that $\mathrm{Dy}^{3+}$ ion has to overcome for entering the spinel crystal lattice resulting in crystal lattice distortion and local strain [11].

3.2. TEM Study. Few representative transmission electron micrographs of the dysprosium doped cobalt ferrite nanoparticles at the sintering temperature of $700^{\circ} \mathrm{C}$ are shown in Figure 2. The average particle sizes are 49, 40, 43 and $37 \mathrm{~nm}$ for the concentration of $x=0.00,0.05,0.10$ and 0.15 , respectively (Table 2 ). In the present case the particle 

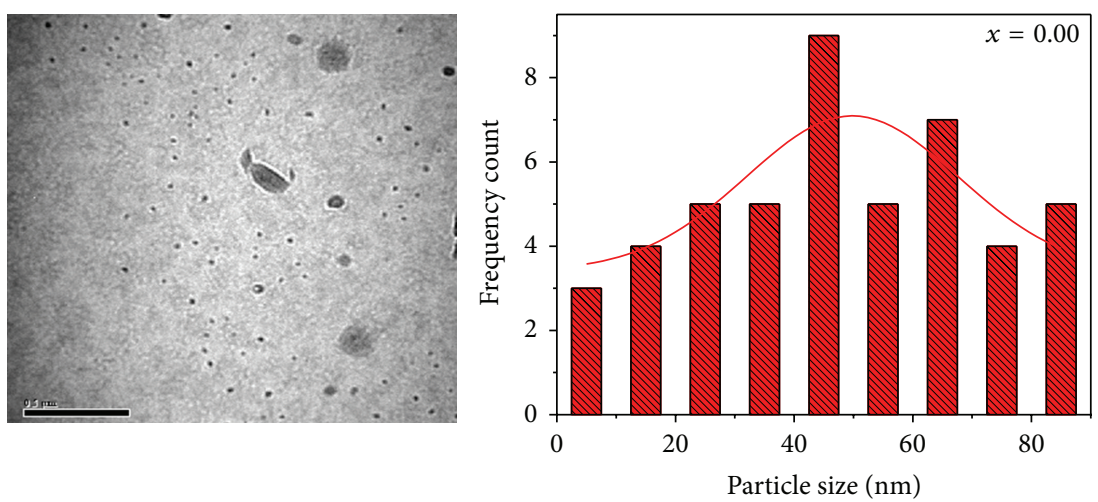

(a)
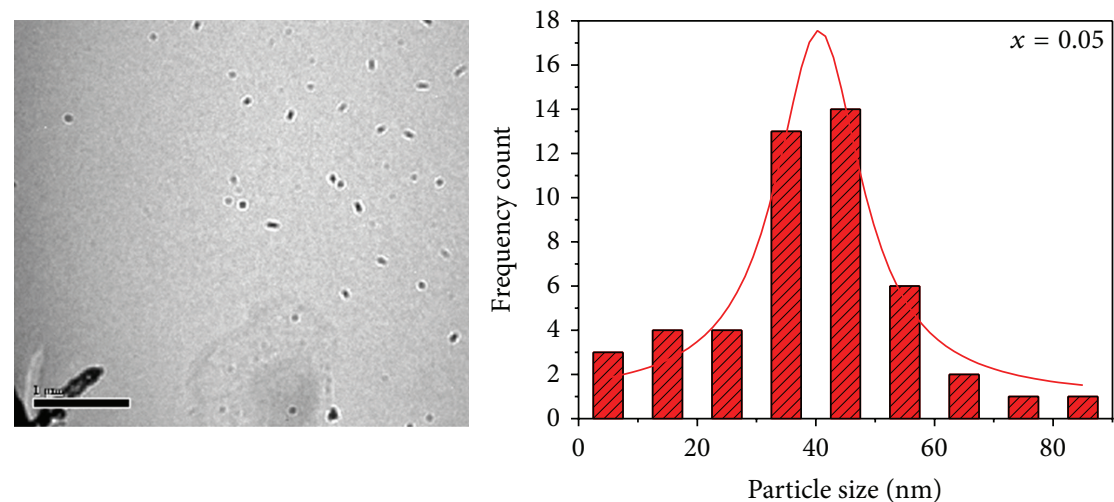

(b)
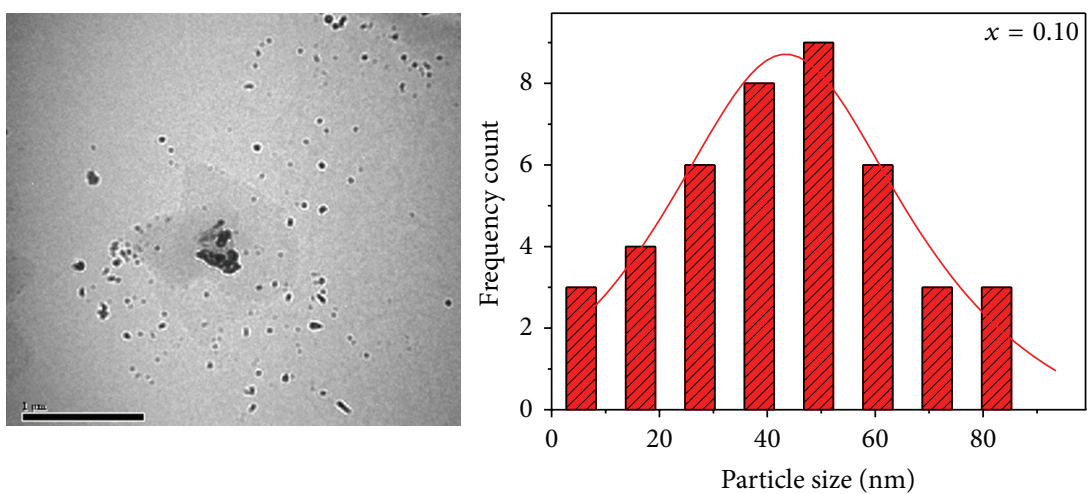

(c)
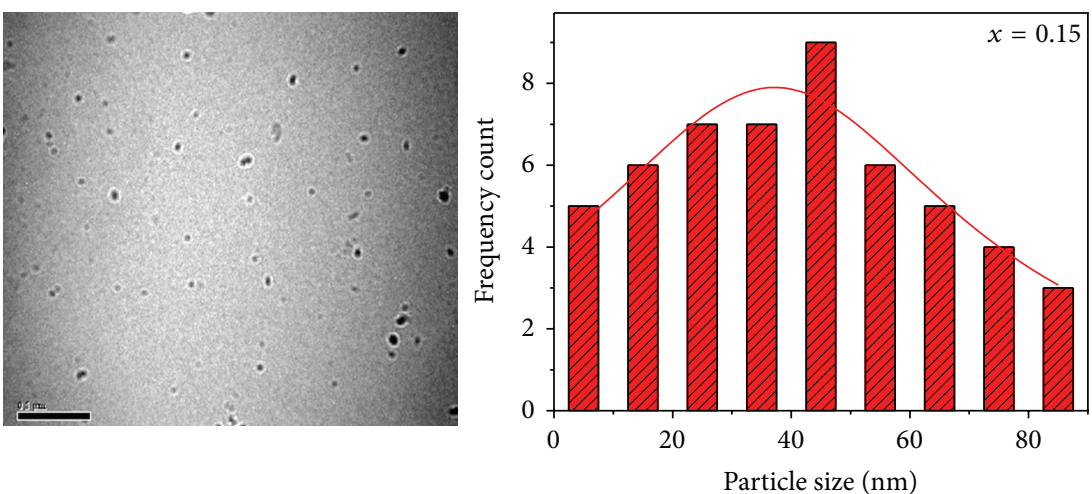

(d)

FiguRE 2: TEM micrographs of $\mathrm{CoFe}_{2-x} \mathrm{Dy}_{x} \mathrm{O}_{4}$ sintered at $700^{\circ} \mathrm{C}$. (a) $x=0.00$; (b) $x=0.05$; (c) $x=0.10$; (d) $x=0.15$. 


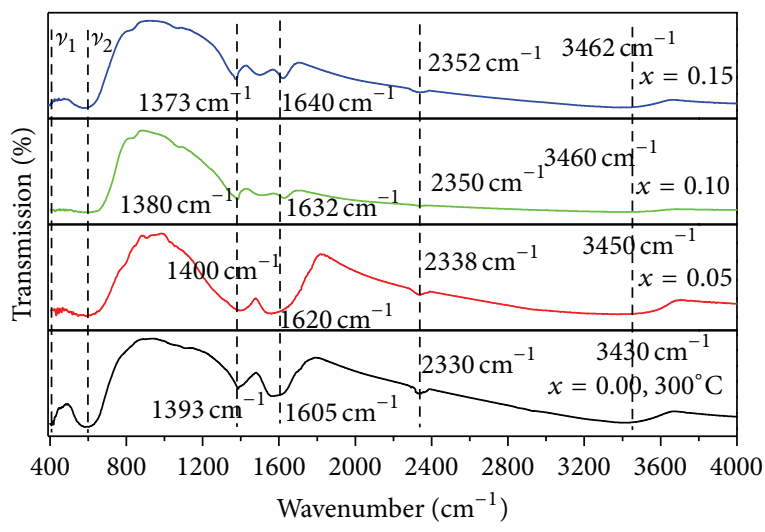

(a)

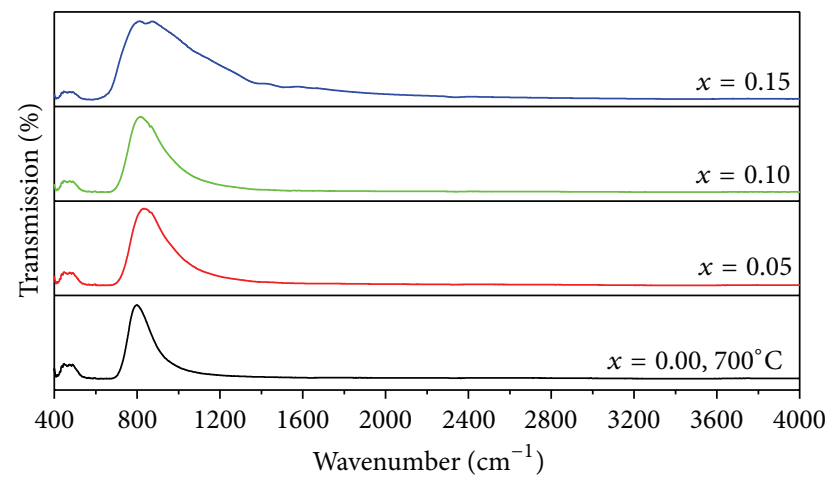

(c)

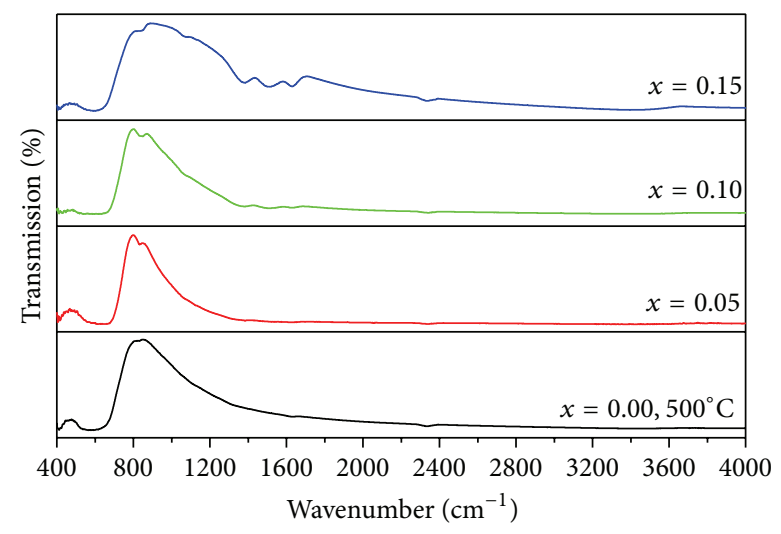

(b)

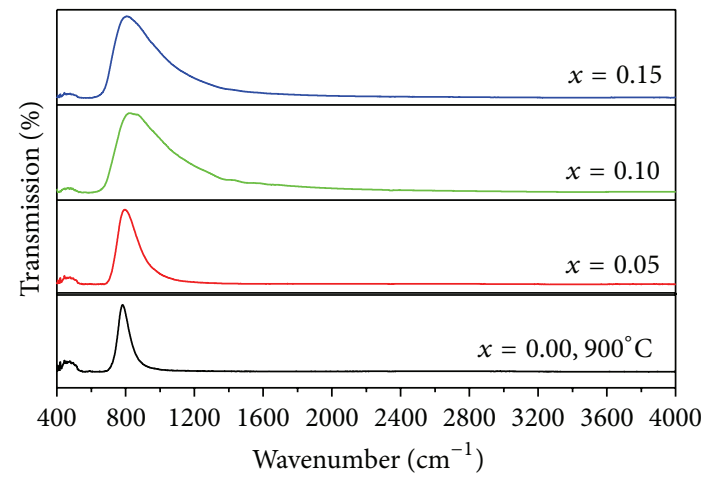

(d)

FIGURE 3: FTIR spectra of $\mathrm{CoFe}_{2-x} \mathrm{Dy}_{x} \mathrm{O}_{4}(x=0.00,0.05,0.10 \& 0.15)$ sintered at different temperatures of (a) $300^{\circ} \mathrm{C},(\mathrm{b}) 500^{\circ} \mathrm{C},(\mathrm{c}) 700^{\circ} \mathrm{C}$, and (d) $900^{\circ} \mathrm{C}$.

TABLE 2: Particle size of samples $\mathrm{CoFe}_{2-x} \mathrm{Dy}_{x} \mathrm{O}_{4}$ sintered at $700^{\circ} \mathrm{C}$.

\begin{tabular}{lc}
\hline Samples & Particle size $(\mathrm{nm})$ \\
\hline$x=0.00$ & 49 \\
$x=0.05$ & 40 \\
$x=0.10$ & 43 \\
$x=0.15$ & 37 \\
\hline
\end{tabular}

size is more than that of the crystallite size, indicating that the particles of the synthesized samples consist of several crystallites. A decreasing trend of particle size was observed with the increase of $\mathrm{Dy}^{3+}$ concentration. Similar behaviour of particle size was observed by other workers also [12].

3.3. FTIR Study. FTIR spectra of dysprosium doped cobalt ferrite which were synthesized at different sintering temperatures $\left(300-900^{\circ} \mathrm{C}\right)$ were recorded in the range of $400-$ $5000 \mathrm{~cm}^{-1}$ at room temperature. The obtained results are shown in Figures 3(a)-3(d). In ferrites, the band appearing at the higher wave number $\left(\nu_{1}, 500-600 \mathrm{~cm}^{-1}\right)$ is assigned to the tetrahedral complexes, while the band appearing at lower wave number $\left(\nu_{2}, 400-450 \mathrm{~cm}^{-1}\right)$ is assigned to the octahedral complexes. The higher wave number $\nu_{1}$ represents the vibration of $\mathrm{Fe}^{3+}-\mathrm{O}^{2-}$ in the sublattice site $\mathrm{A}$, while the lower wave number band $v_{2}$ represents the trivalent metaloxygen vibration at the octahedral B-sites. The difference in the $v_{1}$ and $v_{2}$ band positions is expected because of the difference in the $\mathrm{Fe}^{3+}-\mathrm{O}^{2-}$ distance for the octahedral and the tetrahedral sites [13]. It is seen that the FTIR spectra of the dysprosium doped cobalt ferrite nanoparticles show no residual organic compounds and confirm the formation of the organic free dysprosium doped cobalt ferrite above the sintering temperature of $500^{\circ} \mathrm{C}$. At lower sintering temperature $\left(300^{\circ} \mathrm{C}\right)$ of dysprosium doped cobalt ferrite, the observed peak at about $3410 \mathrm{~cm}^{-1}$ is attributed to the stretching vibration of the hydroxyl group and the other observed peaks at around 1400 and $1600 \mathrm{~cm}^{-1}$ are attributed to the symmetric and asymmetric stretching vibration of $\mathrm{CO}_{2}$ groups. The observed peak around $2330-2358 \mathrm{~cm}^{-1}$ is assigned to the aliphatic and aromatic $\mathrm{C}-\mathrm{H}$ bond stretching. These bands start removing for higher sintering temperature due to the evaporation of $\mathrm{CO}_{2}$ [14]. The ratio of the line position of FTIR bands can be determined by [15] $\nu_{1} / \nu_{2}=\left(K_{t} / K_{0}\right) \sqrt{2}$ where $K_{t}$ and $K_{0}$ are the force constants for the M-O bonds in tetrahedral and octahedral sites, respectively. Deviation of the ratio $K_{t} / K_{0}$ from unity indicates that the expansion at one site is not equally compensated by the shrinkage at another site. In the present case the peak around $800 \mathrm{~cm}^{-1}$ becomes more 
TABLE 3: FTIR data of $\mathrm{CoFe}_{2-\mathrm{x}} \mathrm{Dy}_{\mathrm{x}} \mathrm{O}_{4}$ samples sintered at $300,500,700$, and $900^{\circ} \mathrm{C}$.

\begin{tabular}{|c|c|c|c|c|c|c|c|c|}
\hline \multirow{2}{*}{ Samples } & \multicolumn{2}{|c|}{$300^{\circ} \mathrm{C}$} & \multicolumn{2}{|c|}{$500^{\circ} \mathrm{C}$} & \multicolumn{2}{|c|}{$700^{\circ} \mathrm{C}$} & \multicolumn{2}{|c|}{$900^{\circ} \mathrm{C}$} \\
\hline & $v_{1}\left(\mathrm{~cm}^{-1}\right)$ & $v_{2}\left(\mathrm{~cm}^{-1}\right)$ & $v_{1}\left(\mathrm{~cm}^{-1}\right)$ & $v_{2}\left(\mathrm{~cm}^{-1}\right)$ & $v_{1}\left(\mathrm{~cm}^{-1}\right)$ & $v_{2}\left(\mathrm{~cm}^{-1}\right)$ & $v_{1}\left(\mathrm{~cm}^{-1}\right)$ & $v_{2}\left(\mathrm{~cm}^{-1}\right)$ \\
\hline$x=0.00$ & 567 & 403 & 553 & 410 & 567 & 410 & 560 & 417 \\
\hline$x=0.05$ & 574 & 417 & 581 & 411 & 574 & 417 & 581 & 417 \\
\hline$x=0.10$ & 581 & 424 & 594 & 430 & 584 & 417 & 587 & 424 \\
\hline$x=0.15$ & 594 & 424 & 595 & 437 & 587 & 424 & 594 & 430 \\
\hline
\end{tabular}

TABLE 4: Dielectric constant of $\mathrm{CoFe}_{2-x} \mathrm{Dy}_{x} \mathrm{O}_{4}(x=0.00,0.05,0.10$, and 0.15$)$ at different sintering temperatures.

\begin{tabular}{|c|c|c|c|c|c|}
\hline \multirow{2}{*}{ Samples } & \multirow{2}{*}{ Frequency } & \multicolumn{4}{|c|}{ Dielectric constant $\left(\varepsilon^{\prime}\right)$ at different sintering temperatures } \\
\hline & & $300^{\circ} \mathrm{C}$ & $500^{\circ} \mathrm{C}$ & $700^{\circ} \mathrm{C}$ & $900^{\circ} \mathrm{C}$ \\
\hline \multirow{3}{*}{$x=0.00$} & $75 \mathrm{kHz}$ & 180 & 1127 & 834 & 344 \\
\hline & $1 \mathrm{MHz}$ & 177 & 1370 & 1017 & 385 \\
\hline & $5 \mathrm{MHz}$ & 173 & 1559 & 1143 & 473 \\
\hline \multirow{3}{*}{$x=0.05$} & $75 \mathrm{kHz}$ & 248 & 391 & 567 & 149 \\
\hline & $1 \mathrm{MHz}$ & 246 & 273 & 567 & 149 \\
\hline & $5 \mathrm{MHz}$ & 241 & 242 & 557 & 146 \\
\hline \multirow{3}{*}{$x=0.10$} & $75 \mathrm{kHz}$ & 495 & 293 & 267 & 220 \\
\hline & $1 \mathrm{MHz}$ & 297 & 282 & 263 & 220 \\
\hline & $5 \mathrm{MHz}$ & 276 & 274 & 257 & 216 \\
\hline \multirow{3}{*}{$x=0.15$} & $75 \mathrm{kHz}$ & 245 & 160 & 183 & 692 \\
\hline & $1 \mathrm{MHz}$ & 160 & 151 & 180 & 679 \\
\hline & $5 \mathrm{MHz}$ & 171 & 146 & 183 & 665 \\
\hline
\end{tabular}

and more narrower with increasing sintering temperature, which is due to the grain growth of the particles with increase in sintering temperature. The band positions of dysprosium doped cobalt ferrite sintered at $300^{\circ} \mathrm{C}$ are in the range of $567 \mathrm{~cm}^{-1}$ to $594 \mathrm{~cm}^{-1}$ for $\nu_{1}$ and $403 \mathrm{~cm}^{-1}$ to $424 \mathrm{~cm}^{-1}$ for $v_{2}$. The band positions of the samples sintered $500^{\circ} \mathrm{C}$ are in the range of $553 \mathrm{~cm}^{-1}$ to $595 \mathrm{~cm}^{-1}$ for $\nu_{1}$ and $410 \mathrm{~cm}^{-1}$ to $437 \mathrm{~cm}^{-1}$ for $\nu_{2}$. The band positions of the samples sintered $700^{\circ} \mathrm{C}$ are in the range $567 \mathrm{~cm}^{-1}$ to $587 \mathrm{~cm}^{-1}$ for $\nu_{1}$ and $410 \mathrm{~cm}^{-1}$ to $424 \mathrm{~cm}^{-1}$ for $v_{2}$. The band positions of the samples sintered $900^{\circ} \mathrm{C}$ are in the range of $560 \mathrm{~cm}^{-1}$ to $594 \mathrm{~cm}^{-1}$ for $\nu_{1}$ and $417 \mathrm{~cm}^{-1}$ to $430 \mathrm{~cm}^{-1}$ for $\nu_{2}$. The vibrational band positions for all the samples are shown in Table 3. It is observed that substitution of $\mathrm{Fe}^{3+}$ by $\mathrm{Dy}^{3+}$ causes shifting of band positions $\left(\mathrm{Fe}^{3+}-\mathrm{O}^{2-}\right)$ towards higher frequency side due to increased bond length at $\mathrm{B}$-sites and lattice distortion. This also suggests the occupancy of $\mathrm{Dy}^{3+}$ at B-sites.

3.4. Dielectric Behaviour. The dielectric constant $\varepsilon^{\prime}$ of dysprosium doped cobalt $\left(\mathrm{CoFe}_{2-x} \mathrm{Dy}_{x} \mathrm{O}_{4}\right)$ ferrite has been estimated from capacitance, $C$, of the pellets using the formula given below [16]:

$$
\varepsilon^{\prime}=\frac{C d}{\varepsilon_{0} A}
$$

where $d$ is the thickness of the pellets, $A$ is the crosssectional area in $\mathrm{m}^{2}$, and $\varepsilon_{0}$ is the permittivity of free space. The variation of dielectric constant against frequency for different samples is shown in Figure 4. The values of dielectric constant are shown in Table 4 . The dielectric constant of the dysprosium doped cobalt ferrite $\left(\mathrm{CoFe}_{2-x} \mathrm{Dy}_{x} \mathrm{O}_{4}\right)$ in general decreases with the increase in the frequency. However, in the present case some deviation was observed from this usual behaviour. For the sample sintered at $300^{\circ} \mathrm{C}$, the dielectric constant is almost unchanged with the frequency at the particular concentrations of $x=0.00$ and 0.05 . Similar result is obtained for the sample sintered at $500^{\circ} \mathrm{C}(x=0.10,0.15)$, $700^{\circ} \mathrm{C}(x=0.05,0.10$, and 0.15$)$, and $900^{\circ} \mathrm{C}(x=0.05,0.10)$. An increase in the dielectric constant was observed for the undoped cobalt ferrite sintered at 500,700 , and $900^{\circ} \mathrm{C}$. The decrease of dielectric constant with increasing frequency is due to the fact that above certain frequency of the electric field, the electron exchange between $\mathrm{Fe}^{2+}$ and $\mathrm{Fe}^{3+}$ ion cannot follow the variation of electric field. The space charge carriers in a dielectric material require a finite time to line up their axes parallel to the direction of the applied electric field. If the frequency of the field increases, a point is reached when the space charge carriers cannot cope up with the applied field. As a result the dielectric constant of these materials decreases. As the frequency of the field continuously increases, at some stage the space charge polarization would barely have started 

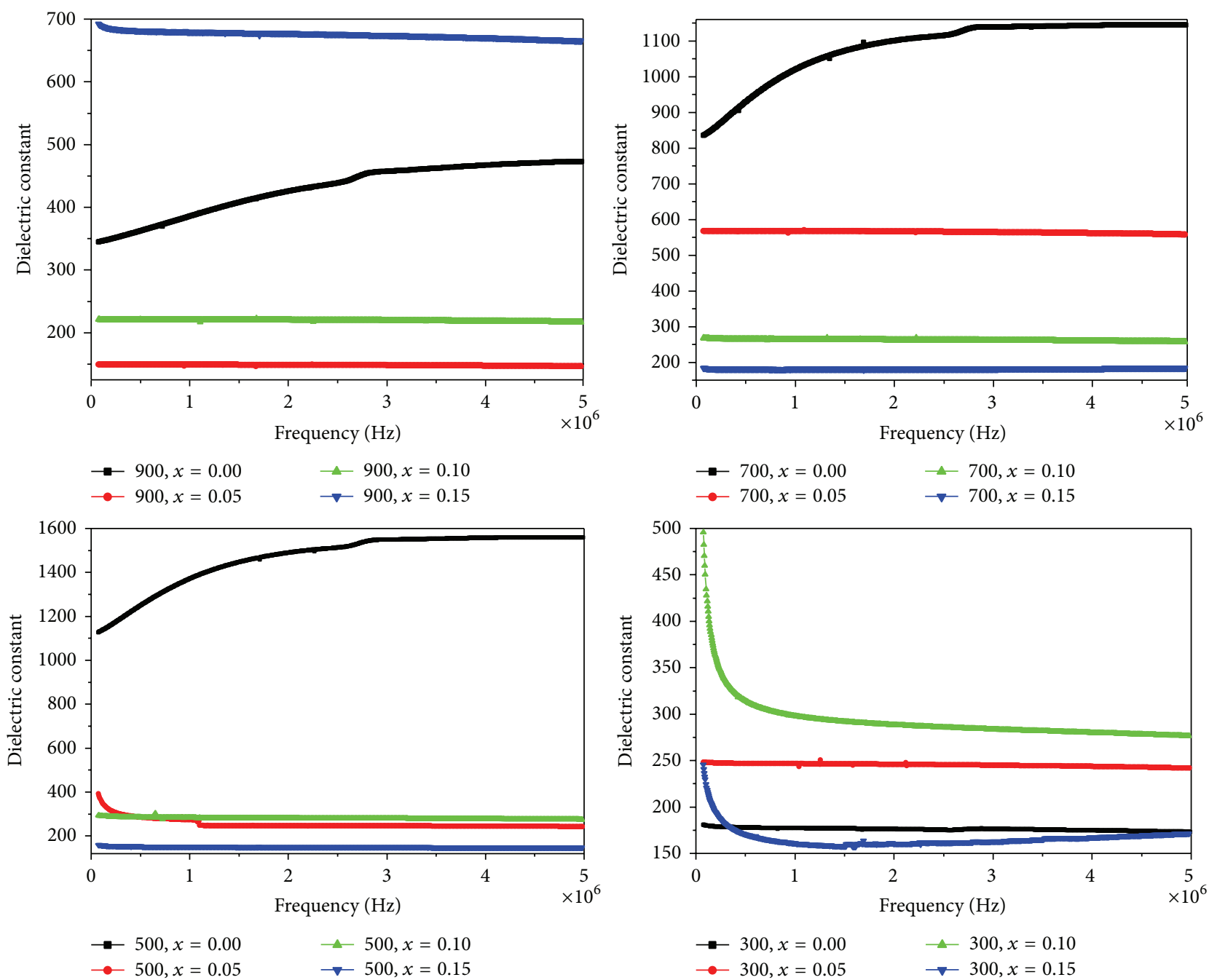

Figure 4: Dielectric constant of $\mathrm{CoFe}_{2-x} \mathrm{Dy}_{x} \mathrm{O}_{4}(x=0.00,0.05,0.10 \& 0.15)$ sintered at $300,500,700$, and $900^{\circ} \mathrm{C}$.

TABLE 5: Dielectric loss tangent $(\tan \delta)$ of $\mathrm{CoFe}_{2-x} \mathrm{Dy}_{x} \mathrm{O}_{4}(x=0.00,0.05,0.10$ and 0.15$)$ at different sintering temperatures.

\begin{tabular}{|c|c|c|c|c|c|}
\hline \multirow{2}{*}{ Samples } & \multirow{2}{*}{ Frequency } & \multicolumn{4}{|c|}{ Dielectric tangent loss $(\tan \delta)$ at different sintering temperatures } \\
\hline & & $300^{\circ} \mathrm{C}$ & $500^{\circ} \mathrm{C}$ & $700^{\circ} \mathrm{C}$ & $900^{\circ} \mathrm{C}$ \\
\hline \multirow{3}{*}{$x=0.00$} & $75 \mathrm{kHz}$ & 0.037 & 0.045 & 0.034 & 0.028 \\
\hline & $1 \mathrm{MHz}$ & 0.005 & 0.158 & 0.151 & 0.159 \\
\hline & $5 \mathrm{MHz}$ & 0.001 & 0.072 & 0.067 & 0.118 \\
\hline \multirow{3}{*}{$x=0.05$} & $75 \mathrm{kHz}$ & 0.010 & 1.79 & 0.0005 & 0.0002 \\
\hline & $1 \mathrm{MHz}$ & 0.001 & 0.253 & 0.0002 & 0.0001 \\
\hline & $5 \mathrm{MHz}$ & 0.001 & 0.0136 & 0.0001 & 0.001 \\
\hline \multirow{3}{*}{$x=0.10$} & $75 \mathrm{kHz}$ & 2.006 & 0.195 & 0.041 & 0.0009 \\
\hline & $1 \mathrm{MHz}$ & 0.343 & 0.022 & 0.00481 & 0.0007 \\
\hline & $5 \mathrm{MHz}$ & 0.073 & 0.005 & 0.003 & 0.0006 \\
\hline \multirow{3}{*}{$x=0.15$} & $75 \mathrm{kHz}$ & 1.644 & 0.159 & 0.051 & 0.0395 \\
\hline & $1 \mathrm{MHz}$ & 0.222 & 0.026 & 0.00481 & 0.00456 \\
\hline & $5 \mathrm{MHz}$ & 0.142 & 0.001 & 0.003 & 0.0107 \\
\hline
\end{tabular}



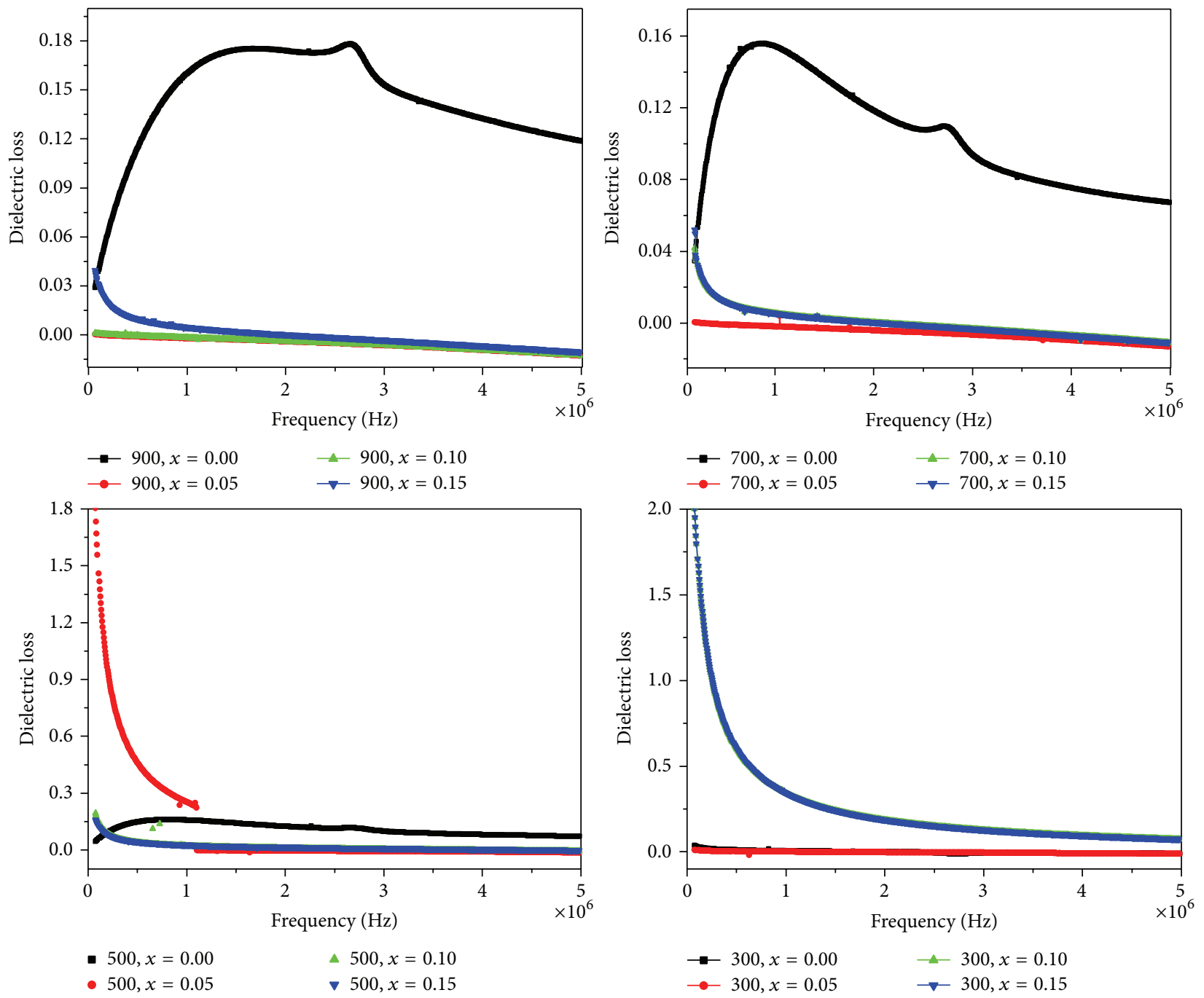

Figure 5: Dielectric loss in $\mathrm{CoFe}_{2-x} \mathrm{Dy}_{x} \mathrm{O}_{4}(x=0.00,0.05,0.10 \& 0.15)$ sintered at 300, 500, 700, and $900^{\circ} \mathrm{C}$.

TABLE 6: Electrical resistivity $(\rho)$ of $\mathrm{CoFe}_{2-x} \mathrm{Dy}_{x} \mathrm{O}_{4}(x=0.00,0.05,0.10$, and 0.15$)$ at different sintering temperatures.

\begin{tabular}{lcccc}
\hline Samples & \multicolumn{3}{c}{ Electrical resistivity $(\Omega$-cm $)$ at different sintering temperatures } \\
& $300^{\circ} \mathrm{C}$ & $500^{\circ} \mathrm{C}$ & $700^{\circ} \mathrm{C}$ & $900^{\circ} \mathrm{C}$ \\
\hline$x=0.00$ & $1.76 \times 10^{7}$ & $5.87 \times 10^{7}$ & $8.07 \times 10^{6}$ & $1.88 \times 10^{6}$ \\
$x=0.05$ & $6.83 \times 10^{7}$ & $1.8 \times 10^{5}$ & $7.87 \times 10^{8}$ & $1.62 \times 10^{9}$ \\
$x=0.10$ & $6.26 \times 10^{7}$ & $6.31 \times 10^{5}$ & $4.95 \times 10^{6}$ & $1.16 \times 10^{8}$ \\
$x=0.15$ & $12.45 \times 10^{7}$ & $25.68 \times 10^{5}$ & $24.37 \times 10^{6}$ & $6.84 \times 10^{6}$ \\
\hline
\end{tabular}

to move before the field reverses and makes virtually no contribution to the polarization and hence to dielectric. The increase in dielectric constant with frequency may be due to resonance effect. The resonance may arise due to the matching of the frequency of charge transfer between $\mathrm{Fe}^{2+} \leftrightarrow \mathrm{Fe}^{3+}$ ions and that of the applied electric field. Similar increase in the dielectric constant was obtained for the La doped cobalt ferrite $[8,17,18]$. The change in dielectric constant with increasing dysprosium concentration may be due to the migration of the $\mathrm{Fe}^{3+}$ ions from octahedral site to tetrahedral site. A similar behaviour was reported in case of the nickel substituted Co-Zn ferrite [6]. It has been reported that dielectric properties of materials are sensitive to the method of synthesis, microstructure, and crystallite size. In the present case, two factors which have dominant effect are sintering temperature and concentration of dopant ions. At lower concentration $\mathrm{Dy}^{3+}$ ions are dissolved in spinel lattice; however, at higher concentration these ions cease 

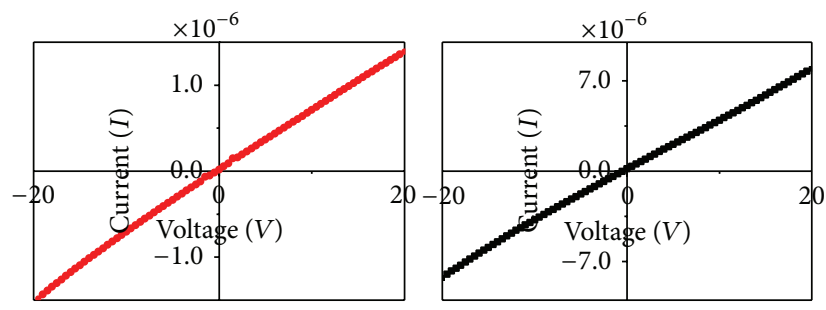

- $300, x=0.05$

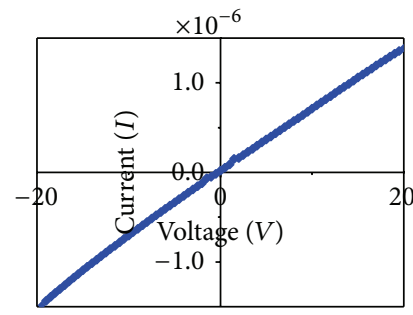

- $300, x=0.15$

- $300, x=0.00$

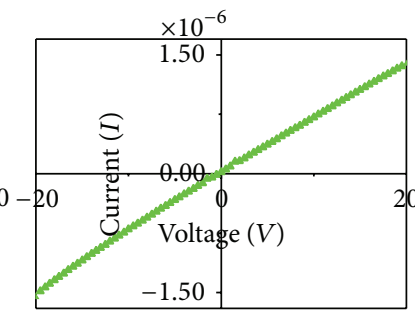

- $300, x=0.10$ (a)
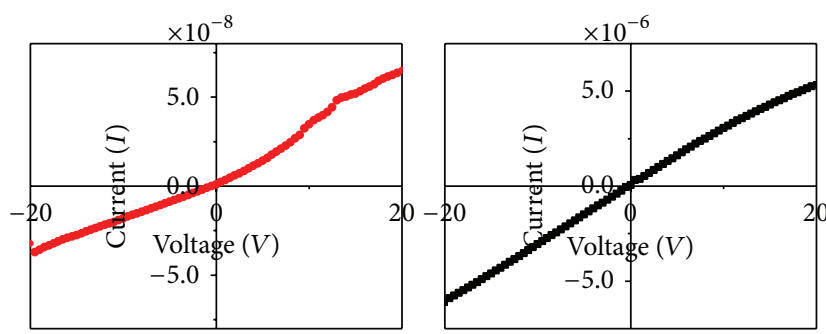

- $700, x=0.00$

- $700, x=0.05$

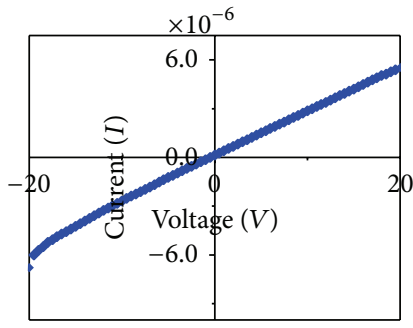

४ 700, $x=0.15$

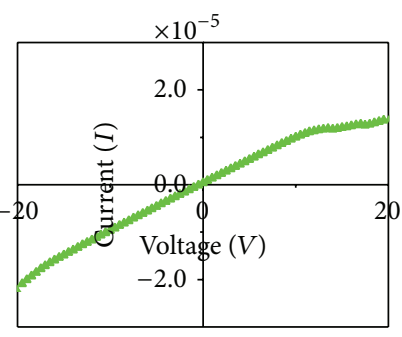

$\triangle 700, x=0.10$
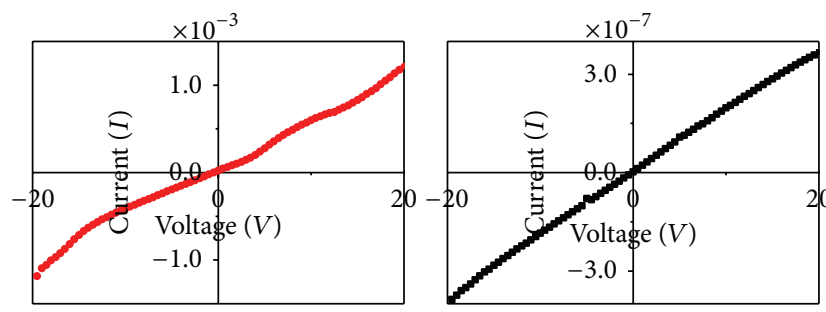

- $500, x=0.05$

- $500, x=0.00$

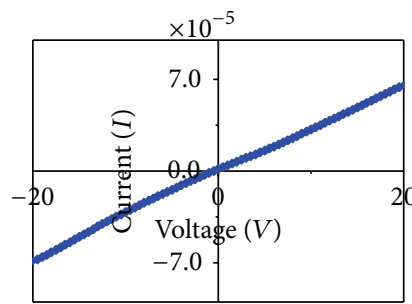

- 500, $x=0.15$

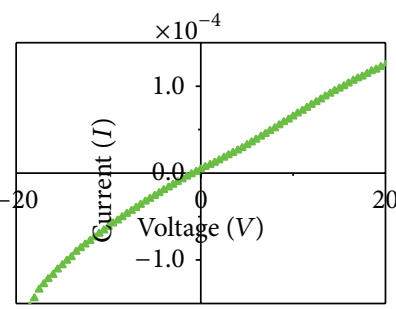

$\triangle 500, x=0.10$

(b)

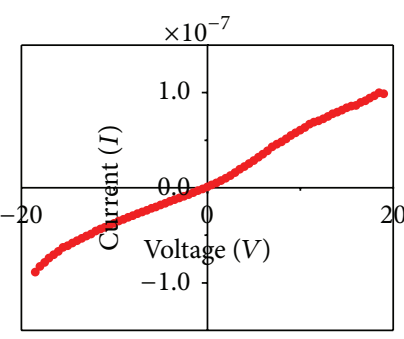

- $900, x=0.05$

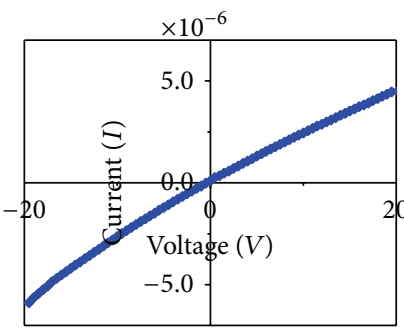

- 900, $x=0.15$

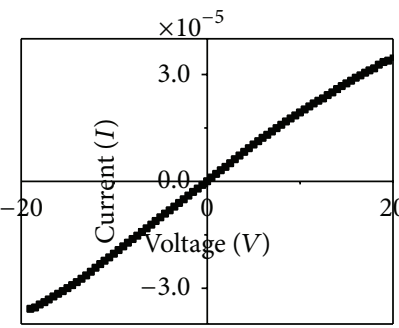

- $900, x=0.00$

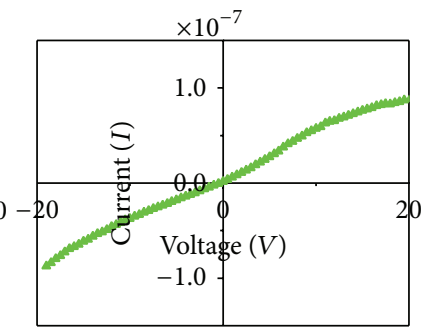

$\triangle 900, x=0.10$

(c)

(d)

Figure 6: $I-V$ curves of $\mathrm{CoFe}_{2-x} \mathrm{Dy}_{x} \mathrm{O}_{4}(x=0.00,0.05,0.10 \& 0.15)$ sintered at different temperatures (a) $300^{\circ} \mathrm{C}$, (b) $500^{\circ} \mathrm{C},(\mathrm{c}) 700^{\circ} \mathrm{C}$, and (d) $900^{\circ} \mathrm{C}$.

to be dissolved, resulting in the presence of $\mathrm{Dy}^{3+}$ ions at grain boundaries at higher dopant concentration. On the other hand low sintering leads to incomplete crystallization; however, crystallization is prominent at higher sintering temperature [19]. The value of dielectric constant in the present case is affected by oxidation state of metallic ions, method of synthesis, and also the solubility of $\mathrm{Dy}^{3+}$ ion. This aspect was studied for nickel ferrite system [20]. Method of synthesis plays an important role in determining the dielectric properties. Synthesis routes which involve comparatively low heat treatment exhibit higher value of dielectric constant and high value of dielectric loss due to presence of organic free radicals and metastable phases. This type of behaviour was studied by Singh et al. [20]. Coprecipitation method requires comparatively higher value of thermal treatment than the nitrate route. This may be one reason for change in the dielectric constant [8]. Another issue is the solubility of rare earth ions in the ferrite matrix. The rare earth ions 
with larger ionic radii have limited solubility in the lattice. The trace amount of the phase which could not be detected by XRD such as the presence of $\mathrm{Dy}^{3+}$ ions on grain boundaries may take part in polarization processes and may cause an increase in the dielectric constant of the materials.

3.5. Dielectric Loss. Dielectric tangent loss $(\tan \delta)$ of $\mathrm{CoFe}_{2-x} \mathrm{Dy}_{x} \mathrm{O}_{4}(x=0.00,0.05,0.10$, and 0.15$)$ was calculated using the relation [16]

$$
\tan \delta=\frac{\varepsilon^{\prime}}{\varepsilon^{\prime \prime}},
$$

where $\varepsilon^{\prime \prime}$ is the imaginary part of the dielectric constant and is a measure of the absorption of energy by the dielectric material from the alternating field, $\delta$ is the loss angle, and $\varepsilon^{\prime}$ is the real part of the dielectric constant. The dielectric loss tangent versus frequency is shown in Figure 5 . The values of the dielectric tangent loss $(\tan \delta)$ at different sintering temperature are tabulated in Table 5. The dielectric tangent loss $(\tan \delta)$ decreases with increase in the frequency and then becomes constant at higher frequencies for each sample. The value of dielectric tangent loss $(\tan \delta)$ depends on a number of factors, such as stoichiometry, $\mathrm{Fe}^{+2}$ content, and structural homogeneity, which in turn depend on the composition and synthesis methods. The decrease in loss tangent with frequency may be attributed to the Maxwell-Wagner polarization [17] and conduction mechanism in ferrites. The dielectric loss tangent $(\tan \delta)$ changes with increase in the dysprosium $\left(\mathrm{Dy}^{3+}\right)$ concentration in cobalt ferrite. An increase in the dielectric loss tangent was observed for the case of undoped cobalt ferrite sintered at 700 and $900^{\circ} \mathrm{C}$.

3.6. I-V Characteristic. The electrical resistivity of the samples was calculated using the formula

$$
\rho=R \frac{A}{L}
$$

where $L$ is the thickness, $R$ is the resistance, and $A$ is the area $\left(A=\pi r^{2}\right)$ of the pellet. Figures $6(\mathrm{a})-6(\mathrm{~d})$ show the $I-V$ characteristics of the samples sintered at different temperatures. The value of electrical resistivity at room temperature is tabulated in Table 6 . The electrical resistivity depends upon the crystal structure and composition of the materials. It changes with the increase in the dysprosium $\left(\mathrm{Dy}^{3+}\right)$ concentration. In the present case of $\mathrm{CoFe}_{2-x} \mathrm{Dy}_{x} \mathrm{O}_{4}$ $(x=0.00,0.05,0.10$, and 0.15$)$ samples, it varies in the range of $1.8 \times 10^{5}$ to $1.62 \times 10^{9} \Omega$-cm for different sintering temperatures. The electrical resistivity of $\mathrm{Mn}-\mathrm{Zn}-\mathrm{Ce}$ ferrites has also been reported in the range of $1.4 \times 10^{5}$ to $4.3 \times$ $10^{8} \Omega-\mathrm{cm}$ [21]. However, other reports show the electrical resistivity in the range $6.4 \times 10^{5}$ to $33.31 \times 10^{6} \Omega-\mathrm{cm}[8]$. The temperature variation of resistivity of these materials is in progress.

\section{Conclusions}

The XRD, TEM, FTIR, dielectric constant, dielectric tangent loss, and electrical behaviour of $\mathrm{CoFe}_{2-x} \mathrm{Dy}_{x} \mathrm{O}_{4}(x=0.00$,
$0.05,0.10$, and 0.15$)$ was studied. In the present case the band positions of FTIR appearing at the higher wave number (i.e., $v_{1}=500-600 \mathrm{~cm}^{-1}$ ) are assigned to the tetrahedral complexes, while lower wave number $\left(\nu_{2}=400-450 \mathrm{~cm}^{-1}\right)$ is assigned to the octahedral complexes. This shows the formation of pure spinel structure of dysprosium doped cobalt ferrite. The dielectric constant of the dysprosium doped cobalt ferrite $\left(\mathrm{CoFe}_{2-x} \mathrm{Dy}_{x} \mathrm{O}_{4}\right)$ in general decreases with the increase in the frequency. However, some deviation was observed from this usual behaviour. For the sample sintered at $300^{\circ} \mathrm{C}$, the dielectric constant is almost unchanged with frequency at the particular concentrations of $x=0.00$ and 0.05. Similar result is obtained for the sample sintered at $500^{\circ} \mathrm{C}(x=0.10,0.15), 700^{\circ} \mathrm{C}(x=0.05,0.10$, and 0.15$)$, and $900^{\circ} \mathrm{C}(x=0.05,0.10)$. The values of electrical resistivity of the materials are in the range of $10^{5}-10^{9} \Omega-\mathrm{cm}$ which may be suitable for the high frequency applications. The high value of dielectric constant is useful in capacitor dielectrics. The low value of dielectric constant is used as microwave absorbers in high frequency applications. In the present study, the different compositions of the materials are suitable for both low and high dielectric constant applications.

\section{Conflict of Interests}

The authors declare that they have no conflict of interests regarding the publication of this paper.

\section{Acknowledgment}

Hemaunt Kumar is thankful to Dr. Mukul Gupta (UGC DAE CSR Indore Centre) for the keen interest and suggestions in XRD measurements and also thankful to Dr. Ranjan Kumar Singh (Department of Physics, BHU) for providing the FTIR facilities.

\section{References}

[1] S. Gubbala, H. Nathani, K. Koziol, and R. D. K. Misra, "Magnetic properties of nanocrystalline $\mathrm{Ni}-\mathrm{Zn}, \mathrm{Zn}-\mathrm{Mn}$, and $\mathrm{Ni}-$ Mn ferrites synthesized by reverse micelle technique," Physica B: Condensed Matter, vol. 348, no. 1-4, pp. 317-328, 2004.

[2] B. Viswanathan and V. R. K. Murthy, Ferrite Materials: Science and Technology, Narosa, New Delhi, India, 1990.

[3] J.-G. Lee, J. Y. Park, and C. S. Kim, "Growth of ultra-fine cobalt ferrite particles by a sol-gel method and their magnetic properties," Journal of Materials Science, vol. 33, no. 15, pp. 39653968, 1998.

[4] S. N. Okuno, S. Hanshimoto, and K. Inomata, "Preferred crystal orientation of cobalt ferrite thin films induced by ion bombardment during deposition ", Journal of Applied Physics, vol. 71, no. 12, pp. 5926-5929, 1992.

[5] H. R. C. S. Andradea, L. M. Searaa, and N. D. S. Mohallema, "Synthesis and characterization of $\mathrm{BaTiO}_{3} / \mathrm{CoFe}_{2} \mathrm{O}_{4}$ thin films," MRS Proceedings, vol. 1368, 2011.

[6] G. Sathishkumar, C. Venkataraju, and K. Sivakumar, "Synthesis, structural and dielectric studies of nickel substituted cobalt-zinc 
ferrite," Materials Sciences and Applications, vol. 1, pp. 19-24, 2010.

[7] G. D. Dwivedi, K. F. Tseng, C. L. Chan et al., "Signature of ferroelectricity in magnetically ordered Mo-doped $\mathrm{CoFe}_{2} \mathrm{O}_{4}$," Physical Review B: Condensed Matter and Materials Physics, vol. 82, Article ID 134428, 2010.

[8] P. Kumar, S. K. Sharma, and M. Singh, "Effect of $\mathrm{La}^{3+}$ doping on the electric, dielectric and magnetic properties of cobalt ferrite processed by co-precipitation technique," Journal of Alloys and Compounds, vol. 508, no. 1, pp. 115-118, 2010.

[9] E. Pervaiz and I. H. Gul, "Structural, electrical and magnetic studies of $\mathrm{Gd}^{3+}$ doped cobalt ferrite nanoparticles," International Journal of Current Engineering and Technology, vol. 2, pp. 377-387, 2012.

[10] M. L. Kahn and Z. J. Zhang, "Synthesis and magnetic properties of $\mathrm{CoFe}_{2} \mathrm{O}_{4}$ spinel ferrite nanoparticles doped with lanthanide ions," Applied Physics Letters, vol. 78, no. 23, pp. 3651-3653, 2001.

[11] X. Meng, H. Li, J. Chen, L. Mei, K. Wang, and X. Li, "Mössbauer study of cobalt ferrite nanocrystals substituted with rare-earth $\mathrm{Y}^{3+}$ ions," Journal of Magnetism and Magnetic Materials, vol. 321, no. 9, pp. 1155-1158, 2009.

[12] A. T. Raghavender, R. G. Kulkarni, and K. M. Jadhav, "Magnetic properties of mixed cobalt-aluminum ferrite nanoparticles," Chinese Journal of Physics, vol. 48, no. 4, pp. 512-522, 2010.

[13] R. M. Mohamed, M. M. Rashad, F. A. Haraz, and W. Sigmund, "Structure and magnetic properties of nanocrystalline cobalt ferrite powders synthesized using organic acid precursor method," Journal of Magnetism and Magnetic Materials, vol. 322, no. 14, pp. 2058-2064, 2010.

[14] M. Gharagozlou, "Synthesis and characterization of nanocrystalline magnetic pigment via coordinated precursors," Progress in Color, Colorants and Coatings, vol. 2, no. 1, pp. 35-43, 2009.

[15] M. A. Ahmed, E. Ateia, and F. M. Salem, "Spectroscopic and electrical properties of $\mathrm{Mg}$-Ti ferrite doped with different rareearth elements," Physica B: Condensed Matter, vol. 381, no. 1-2, pp. 144-155, 2006.

[16] K. M. Batoo, "Structural and electrical properties of $\mathrm{Cu}$ doped $\mathrm{NiFe}_{2} \mathrm{O}_{4}$ nanoparticles prepared through modified citrate gel method," Journal of Physics and Chemistry of Solids, vol. 72, no. 12, pp. 1400-1407, 2011.

[17] J. C. Maxwell, A Treatise on Electricity and Magnetisim, vol. 1, Oxford University Press, New York, NY, USA, 1973.

[18] K. W. Wanger, "Zur theorie der unvollkommenen dilektrika," Annalen der Physik, vol. 345, no. 5, pp. 817-855, 1913.

[19] J. P. Singh, C. L. Chen, C. L. Dong et al., "Effect of intermediate annealing on the structural, electrical and dielectric properties of zinc ferrite: an XANES investigation," Science of Advanced Materials, vol. 5, no. 2, pp. 171-181, 2013.

[20] J. P. Singh, G. Dixit, R. C. Srivastava, H. M. Agrawal, and K. Asokan, "Looking for the possibility of multiferroism in $\mathrm{NiGd}_{0.04} \mathrm{Fe}_{1.96} \mathrm{O}_{4}$ nanoparticle system," Journal of Physics D: Applied Physics, vol. 44, no. 43, Article ID 435306, 2011.

[21] D. Ravinder and B. R. Kumar, "Electrical conductivity of cerium substituted Mn-Zn ferrites," Materials Letters, vol. 57, no. 11, pp. 1738-1742, 2003. 

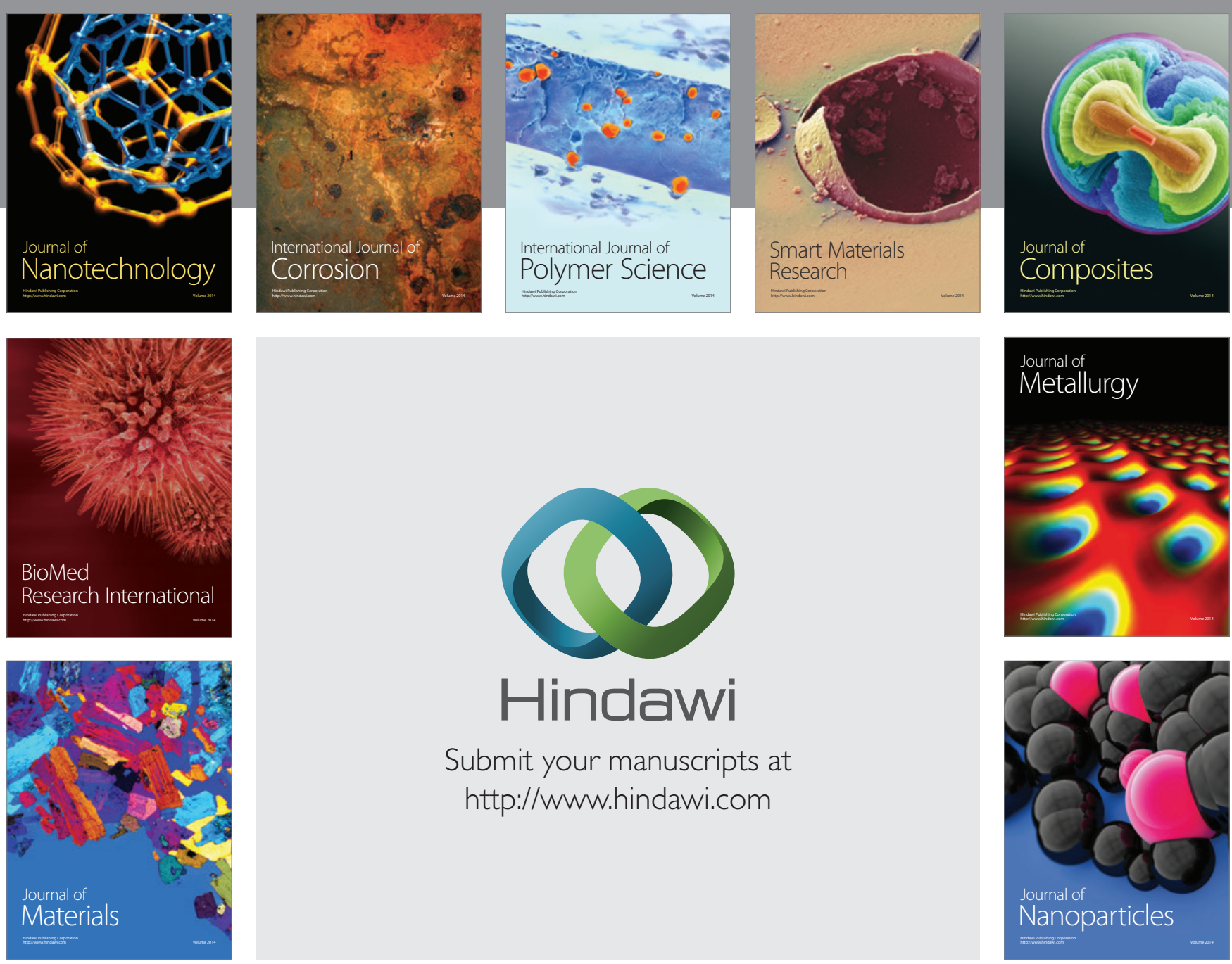

Submit your manuscripts at http://www.hindawi.com
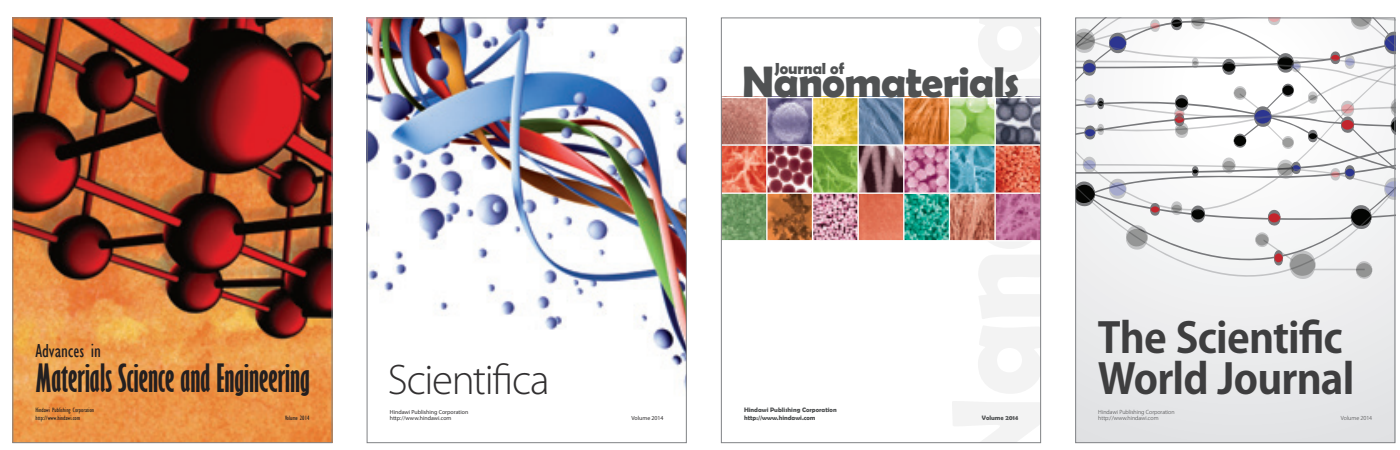

\section{The Scientific World Journal}
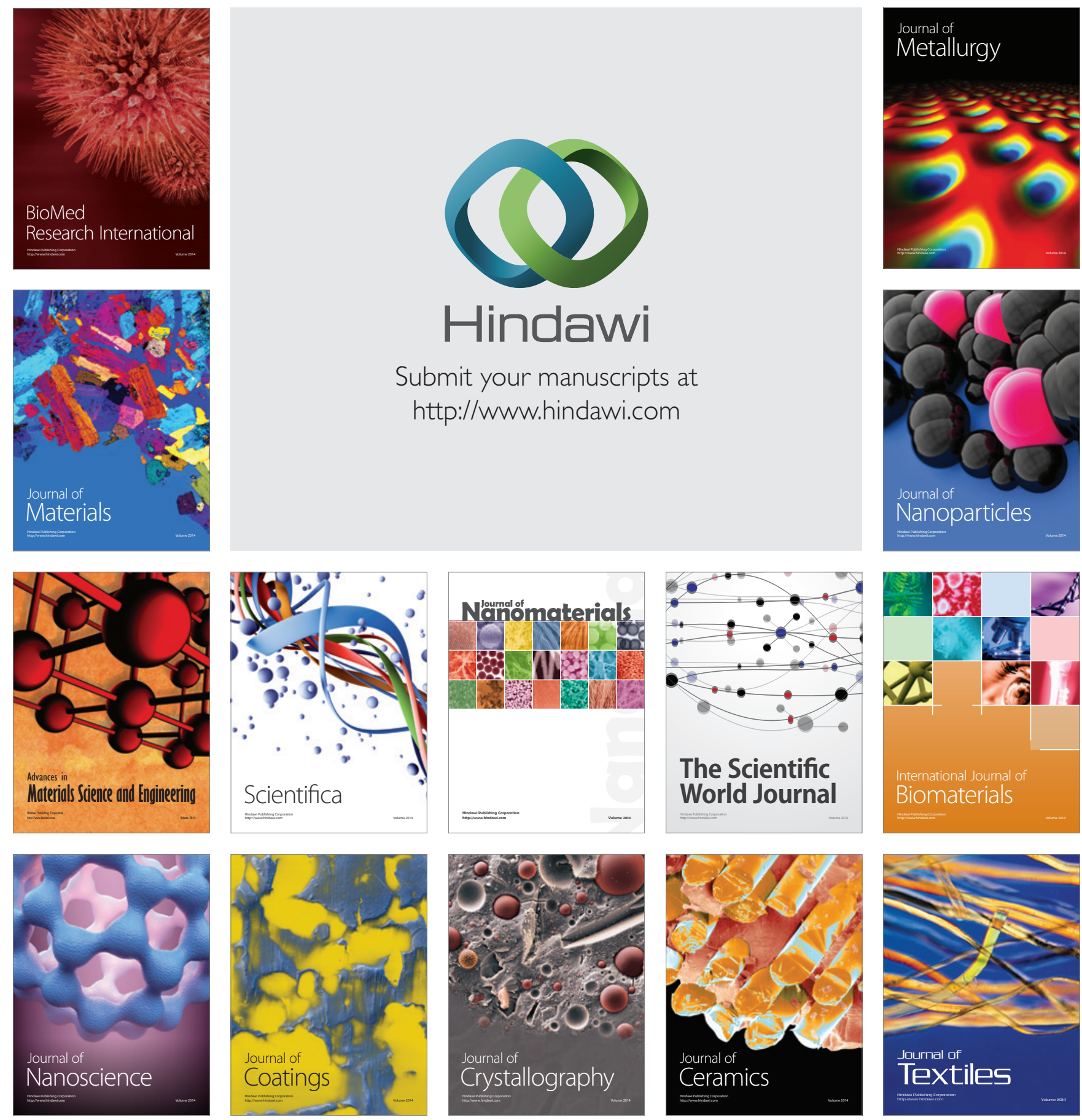